\title{
The Missing Mass Problem
}

\author{
Angel Fierros Palacios \\ División de Energías Alternas, Instituto de Investigaciones Eléctricas, Mexico City, Mexico \\ Email: afierros@iie.org.mx
}

Received 4 June 2016; accepted 21 June 2016; published 24 June 2016

Copyright (C) 2016 by author and Scientific Research Publishing Inc.

This work is licensed under the Creative Commons Attribution International License (CC BY).

http://creativecommons.org/licenses/by/4.0/

(c) (i) Open Access

\section{Abstract}

In this paper a possible solution to the missing mass problem is proposed.

\section{Keywords}

\section{The Missing Mass Problem}

\section{Introduction}

After the red shifts in galaxies' spectral lines were observed in 1912, in 1920-1929 decade it was discovered the evolutionary phenomenon known as the Expansion of the Universe. In the scope of Astronomy, attention was particularly directed toward the beginning and the final fate of the Cosmos; as well as to the proposition of some theoretical models, based on A. Einstein's General Theory of Relativity, in order to explain the behavior of the Universe as a whole. Concerning the beginning of the Universe, G.E. Lemaitre stated an interesting physical theory. He proposed that in the beginning all the galaxies in the Universe were concentrated in a single lamp, which he called the Primeval Atom. This primeval atom then exploded, in the most fantastic and catastrophic explosion called by G. Gamow the Big Bang, flinging the galaxies off into space. The Big Bang sent all the fragments of the primeval atom moving away from one another; and this motion is still visible at present. It was established that the more distant a galaxy, the greater, in direct proportion is its speed of recession, as determined by the shifts of its spectral lines to the longer or red wavelengths. According to Lemaitre, the velocities of recession of those galaxies were in fact, proportional to their distances. This relationship is now known as the law of red shifts, or sometimes Hubble's law [1].

Concerning the final fate of the Universe, two alternatives seem ultimately possible. Either gravity is strong enough so that the universal expansion will slow down, stop, and become a contraction; or the expansion is sufficiently rapid that the rapidly increasing distances between the galaxies will overcome gravity, and the $\boldsymbol{U n}$ iverse will go on expansion forever. The Universe that stops expanding is called a closed Universe, and the ever expanding one is an open Universe. However, there is a borderline case, in which gravity is just strong enough that the distances between any two galaxies increases towards a limiting value, but gravity is not strong enough 
to bring the Universe back together again. On the other hand, the present shape of the Universe is also related to its future if gravity is assumed to be the only force governing its evolution. Apparently, the future of the Universe also depends critically on its curvature, because the curvature in turn depends upon the mean density of matter in space. Anyway, if the Universe is open, it will go expanding forever, whereas if it is closed, its present expansion will eventually cease and be succeeded by a general contraction. In the evolutionary cosmologies the Universe is now expanding. Whether or not the expansion will continue or will eventually stop and give way to contraction, depends on the existence in space of a huge amount of matter. Unfortunately, that amount of matter needed to stop the expansion has not been found yet. This fact is what is called sometimes the missing mass problem.

\section{The Missing Mass}

If some results of Einstein's Special Theory of Relativity are used, it is possible to propose a solution to the missing mass problem. According to Einstein's theory no material particle could possible travel with a velocity greater than the velocity of light in vacuum. Thus it becomes obvious that no material particle could be accelerated beyond the light barrier [2]. This argument still stands. On the other hand, the Universe contains many types of bodies: galaxies, clusters of galaxies, stars, planets, dust and gas, and also many other bodies like black holes and quasars. All of them are bodies which have a proper mass different from zero; so that, are bodies which can only travel at velocities smaller than the velocity of light in the empty space; in such a way that the only way by which they can move is by means of an acceleration process. Moreover, in his original paper on relativity, Einstein said that there is an upper limit of the velocity for the material bodies. In fact, the mass of those bodies would get infinitely large upon approaching the velocity toward the upper limit $c$; as it is easy to see from the following relativistic mass transformation equation.

$$
m=\frac{m_{o}}{\sqrt{1-\frac{v^{2}}{c^{2}}}}
$$

where $m_{o}$ is the proper mass and $m$ is the observed mass of the body which travels at the velocity $v$. An examination of the previous equation, shows that when $v \rightarrow c, m \rightarrow \infty$. Thus as the velocity of a body increases toward the velocity of light in the empty space, the mass of the body increases toward infinity. An infinite mass moving at a very low velocity would have infinite energy. Since it is absurd for any body with finite proper mass mo to have infinite energy, it must conclude that it is impossible for such a body to move with the light velocity in the empty space (see the following graphics); and this is valid for all the bodies contained in the Universe. Thus anyone of them could travel at velocities equal to the light velocity in vacuum. However, the relativistic mass transformation process produces an increase of the inertial mass with velocity; in such a way that this fact seems to be another force capable to stop the expansion and bring the Universe back together again.

Finally, when the Principle of The Equivalence of Gravitation and Inertia is applied, it is easy to see that the gravitational mass needed to slow down and stop the expansion process is just the increase of the inertial mass with velocity of the bodies contained in the Universe. See Figure 1.
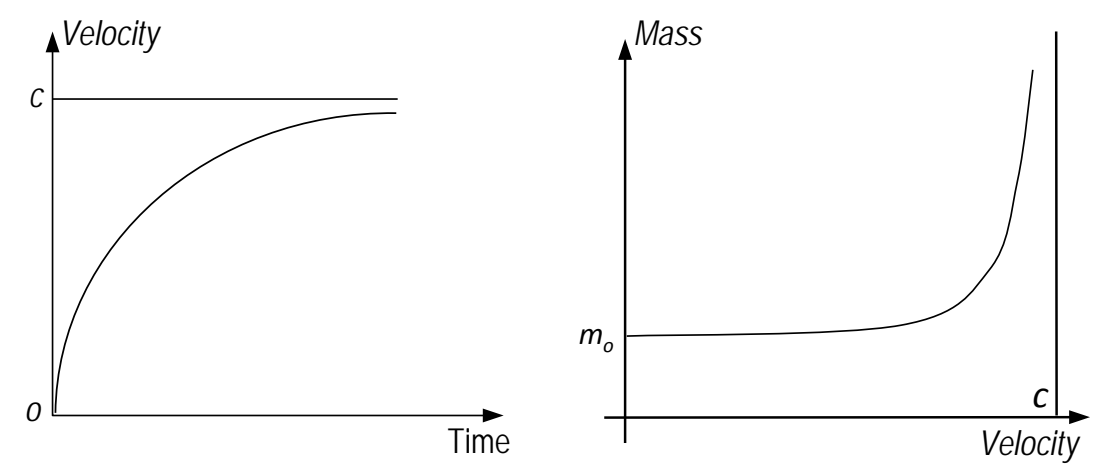

Figure 1. The relativistic velocity, and the relativistic transformation of the mass. 


\section{Conclusions}

The evolution of the Universe was revealed in 1920s when E.P. Hubble extended earlier measurements of the distant galaxies spectra and found that all but a few of the nearest galaxies were speeding away from Earth. Hubble and his colleagues discovered the law that bears Hubble's name: Velocity of recession equals Hubble's constant times distance.

The Universe is expanding now and has been expanding for a long time. Following the expansion backward, it is found that there was a time when all the matter and the energy in the Universe were packed tightly together. The explosion of that Primeval Atom provided impetus for the expansion that it is now observed. The explosion which marked the beginning of the evolution of the Universe is called the Bing Bang Theory. Anyway, if the Universe is open it will go expanding forever, whereas if it is closed the expansion will slow to a stop, and then will become a contraction [3].

According to the previous arguments given in the former paragraph, it seems that the relativistic mass transformation process just produces the missing mass needed to slow down and stop the expansion, in such a way that this is something to be and not to be in the Universe. Moreover, this picture apparently points out toward a closed Universe model.

\section{References}

[1] Weinberg, S. (1972) Gravitation and Cosmology. Principles and Applications of the General Theory of Relativity. John Wiley \& Sons, Inc., New York London Sydney Toronto.

[2] Resnick, R. (1997) Basic Concept in Relativity and Early Quantum Theory. (Versión en español de la Casa Editorial Limusa). John Wiley and Sons, México, España, Venezuela, Colombia.

[3] Harry Shipman, L. (1976) Black Holes, Quasars, \& the Universe. Houghton Mifflin Company, Atlanta Dallas Geneva, Ilinois Hopewell, New Jersey Palo Alto London.

\section{Submit or recommend next manuscript to SCIRP and we will provide best service for you:}

Accepting pre-submission inquiries through Email, Facebook, Linkedin, Twitter, etc A wide selection of journals (inclusive of 9 subjects, more than 200 journals)

Providing a 24-hour high-quality service

User-friendly online submission system

Fair and swift peer-review system

Efficient typesetting and proofreading procedure

Display of the result of downloads and visits, as well as the number of cited articles

Maximum dissemination of your research work

Submit your manuscript at: http://papersubmission.scirp.org/ 